\title{
Extended Dust Shells Surrounding AGB Stars Revealed with ISO
}

\author{
H. Izumiura
}

Okayama Astrophysical Observatory, NAOJ, Kamogata, Asakuchi, Okayama 719-0232, JAPAN

\author{
O. Hashimoto \\ Gunma Astronomical Observatory 1-18-7 Ohotomo, Maebashi, \\ Gunma 371-0847, JAPAN
}

\begin{abstract}
We obtained high resolution far-infrared images of the circumstellar dust shells of several AGB carbon stars using ISOPHOT on board the ISO. We used the C-100 and C-200 detector arrays in PHT32 oversampling mode at $90 \mu \mathrm{m}$ and $160 \mu \mathrm{m}$ except for U Ant, which was observed at $60 \mu \mathrm{m}$ and $90 \mu \mathrm{m}$. AFGL 3068 is very compact in both bands. $\mathrm{R} \mathrm{Scl}$ is marginally resolved at $90 \mu \mathrm{m}$. U Ant shows a double shell structure, a compact but well resolved shell surrounded by a very extended envelope. Y CVn exhibits a very extended, hollow dust shell. We analyse the structure of the dust shells using a simple dust shell model and discuss the mass-loss behaviors, particularly in conjunction with the thermal pulses on the AGB.
\end{abstract}

\section{Introduction}

Low- and intermediate-mass stars are known to undergo intense mass-loss when they are on the asymptotic giant branch (AGB). The mass-loss is considered to be one of the essential processes of the AGB evolution. It is, thus, important to understand when and how the AGB mass-loss occurs in individual stars. Since the AGB mass-loss is thought to be closely related to thermal pulses, it is crucial to trace the mass-loss history on time scales at least comparable to the pulse periods which are predicted to be about $10^{4}-10^{5}$ years. Observing extended dust shells in the far-infrared is the only way to study the mass-loss history on such time scales (Waters et al. 1994; Izumiura et al. 1996, 1997). In this paper we give a brief summary of our mapping observations of AGB carbon stars in the far-infrared and discuss the implications of the results.

\section{Observations}

We have observed stars listed in Table 1 in the far-infrared using ISOPHOT (Lemke et al. 1996) in a Guaranteed-Time program, HIZUMIUR_REDSTAR2 and an open-time program, HIZUMIUR_RYDRA of the Infrared Space Observatory (ISO, Kessler et al. 1996). The fifth column gives the time spent for 
each observation in seconds. In Fig. 1, we show the position of the program stars in the IRAS two-color diagram (cf. van der Veen \& Habing 1988). We

Table 1. Log of observations of program carbon stars

\begin{tabular}{lccrccr}
\hline Object & R.A.(J2000) & Decl.(J2000) & Filter & TDT & Map Size & Date \\
\hline R Scl & 1h 26m 58.0s & $-32^{\circ} 32^{\prime} 34.0^{\prime \prime}$ & 90 & 3462 & $35^{\prime} \times 10^{\prime}$ & 21 Jul 1996 \\
& & & 160 & 3278 & $36^{\prime} \times 14^{\prime}$ & 21 Jul 1996 \\
R For & 2h 29m 15.1s & $-26^{\circ} 05^{\prime} 52.9^{\prime \prime}$ & 90 & 3462 & $35^{\prime} \times 10^{\prime}$ & 5 Feb 1998 \\
& & & 160 & 2998 & $30^{\prime} \times 14^{\prime}$ & 5 Feb 1998 \\
IRC +10216 & 9h 47m 57.4s & $13^{\circ} 16^{\prime} 43.6^{\prime \prime}$ & 90 & 4932 & $50^{\prime} \times 10^{\prime}$ & 8-9 May 1996 \\
& & & 160 & 4818 & $51^{\prime} \times 14^{\prime}$ & 9 May 1996 \\
U Ant & 10h 35m 12.9s & $-39^{\circ} 33^{\prime} 44.7^{\prime \prime}$ & 60 & 2463 & $24^{\prime} \times 10^{\prime}$ & 26 Jul 1996 \\
& & & 90 & 2433 & $24^{\prime} \times 10^{\prime}$ & 26 Jul 1996 \\
Y CVn & 12h 45m 07.8s & $45^{\circ} 26^{\prime} 24.0^{\prime \prime}$ & 90 & 3462 & $35^{\prime} \times 10^{\prime}$ & 25 Apr 1996 \\
& & & 160 & 3278 & $36^{\prime} \times 14^{\prime}$ & 25 Apr 1996 \\
AFGL 3068 & $23 \mathrm{~h} \mathrm{19m} \mathrm{12.4s}$ & \multirow{2}{*}{$17^{\circ} 11^{\prime} 35.4^{\prime \prime}$} & 90 & 3462 & $35^{\prime} \times 10^{\prime}$ & 22 Jun 1996 \\
& & & 160 & 3278 & $36^{\prime} \times 14^{\prime}$ & 22 Jun 1996 \\
\hline RY Dra & 12h 56m 25.7s & $65^{\circ} 59^{\prime} 38.9^{\prime \prime}$ & 90 & 3462 & $35^{\prime} \times 10^{\prime}$ & 12 May 1997 \\
& & & 160 & 3698 & $36^{\prime} \times 14^{\prime}$ & 12 May 1997 \\
\hline
\end{tabular}

used the C-100 and C-200 cameras and the PHT32 astronomical observation template (AOT, Klaas et al. 1994), which provides oversampled maps with the focal plane chopper. We selected the C_60 and C_90 filters for the C-100 camera and C_160 filter for C-200 camera. C-100 and C-200 cameras consist of $3 \times 3$ array of $43^{\prime \prime} .5$ square pixel and $2 \times 2$ array of $89^{\prime \prime} .4$ square pixel, respectively. Oversampling factors in Y- and Z-directions (Klaas et al. 1994) for C-100 were 3 and $2 / 3$, respectively, which resulted in spatial sampling frequencies of $15^{\prime \prime}$ and $23^{\prime \prime}$ with the array configuration. For C-200 the oversampling factors in Yand Z-directions were 3 and 1 , respectively, and the sampling frequencies were $30^{\prime \prime}$ and $92^{\prime \prime}$. C_60, C_90 and C_160 filters are centered at $60.8 \mu \mathrm{m}, 95.1 \mu \mathrm{m}$, and $174 \mu \mathrm{m}$, respectively. In each map the object was placed at the center. The raw data were processed with standard procedures using PHOT Interactive Analysis (PIA, Gabriel et al. 1997) version 7.1 released in 1998. The obtained images were analysed using a simple dust shell model described in Izumiura et al. (1997). In the following we discuss the results on R Scl, U Ant, Y CVn, RY Dra, and AFGL 3068. We do not, however, describe here neither IRC +10216 nor $\mathrm{R}$ For because there are some calibration problems in the data.

\section{AFGL 3068 \& R Scl}

In Fig. 2 we plot the brightness profile of $\mathrm{R} \mathrm{Scl} \mathrm{at} 90 \mu \mathrm{m}$ as well as those of AFGL 3068, IRC -10529, and WX Psc scaled to R Scl. The last two stars are oxygen-rich AGB stars that were also observed in our GT program. The profiles of AFGL 3068, IRC -10529, and WX Psc are almost indistinguishable, indicating that AFGL 3068 is apparently as compact as the two O-rich AGB stars which have been known to possess a very thick and compact dust shell (Hashimoto \& Izumiura 1997). Probably AFGL 3068 is much smaller than the ISOPHOT beam at $90 \mu \mathrm{m}$ for which the FWHM is estimated to be about $50^{\prime \prime}$. 


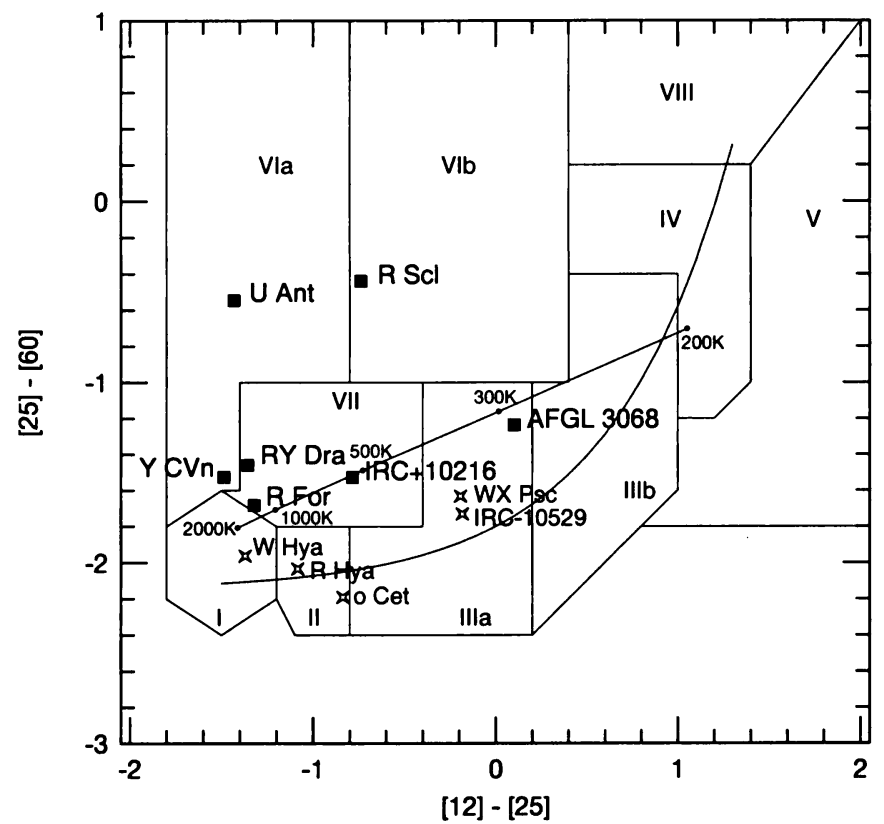

Figure 1. Location of observed carbon stars (filled squares) on the IRAS two-color diagram. Oxygen-rich AGB stars observed in our program are also shown (crosses)

No obvious extended emission is present either around this star. We have found, however, that $\mathrm{R} \mathrm{Scl}$ is marginally resolved with the ISOPHOT beam at $90 \mu \mathrm{m}$. Besides, a hollow dust shell is postulated in order to reproduce the observed brightness profile on the basis of our dust shell model. The apparent inner radius is estimated to be about $11^{\prime \prime}-16^{\prime \prime}$, and the corresponding shell thickness, which is less well determined, to be about $37^{\prime \prime}-12^{\prime \prime}$. The hollow dust shell is consistent with the optical dust shell (Izumiura et al. 1998) as well as with the gas shell seen in mm-wave CO lines (Olofsson et al. 1996). The inner radius of the dust shell is also consistent with those of the $\mathrm{CO}$ and optical shells. The mass-loss rate in the dust shell has been estimated to be around $10^{-6} \mathrm{M}_{\odot} \mathrm{yr}^{-1}$ for a reasonable set of stellar parameters. $\mathrm{R} \mathrm{Scl}$ is, however, not associated with a very extended dust shell like the one seen in Y CVn. This is an important result since this star is one of the most likely candidates for a multiple shell source if every thermal pulse on the AGB gives rise to a significant modulation of mass-loss. We will further discuss this later.

\section{U Ant}

In Fig. 3, we show the $90 \mu \mathrm{m}$ map of $\mathrm{U}$ Ant in an intensity scale which emphasizes faint extended emission. The image shows a central bright component of $100^{\prime \prime} \mathrm{FWHM}$ surrounded by a very extended, faint emission component with $10^{\prime}$ diameter. The central component is well resolved by the ISOPHOT beam 


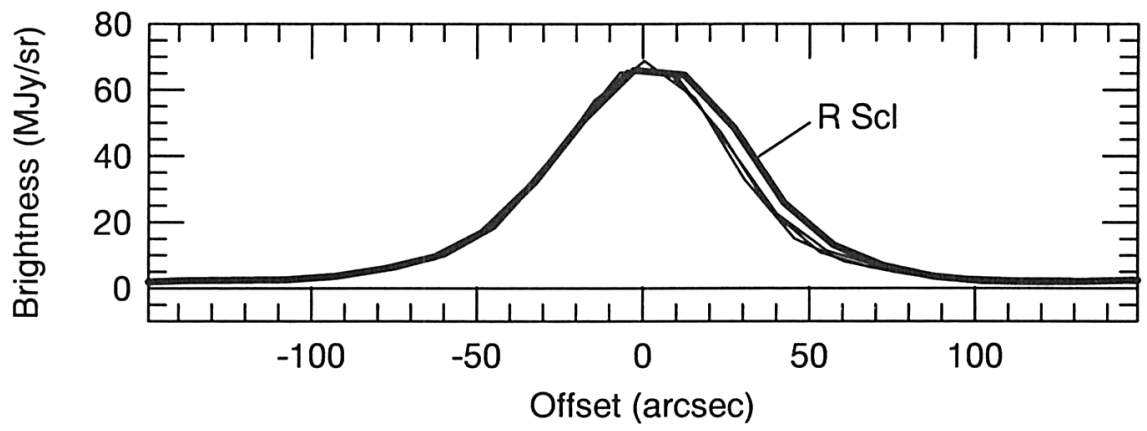

Figure 2. Brightness profile of $\mathrm{R} \mathrm{Scl}$ at $90 \mu \mathrm{m}$ (thick line), and those of AFGL 3068, IRC -10529, and WX Psc scaled to R Scl (thin lines)

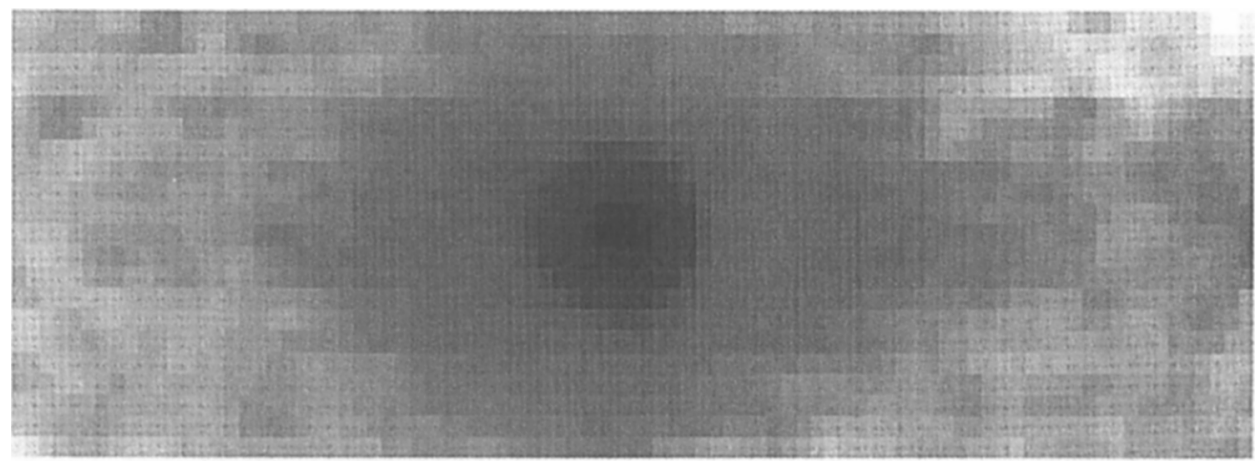

Figure 3. ISOPHOT image of $\mathrm{U}$ Ant at $90 \mu \mathrm{m}$, which measures $1155^{\prime \prime} \times 483^{\prime \prime}$ with a pixel size of $15^{\prime \prime} \times 23^{\prime \prime}$

(cf. Fig. 2). The two emission components are also observed in the $60 \mu \mathrm{m}$ image. On the basis of our model analysis it turns out necessary to introduce at least two concentric, hollow dust shells in order to reproduce the observed brightness distribution. The inner radii of the two shells are well determined as $39^{\prime \prime}$ and $170^{\prime \prime}$. The outer radii are difficult to determine, but we find necessary to place a significant gap between the outer radius of the inner shell and the inner radius of the outer shell, i.e. the outer shell is detached from the inner shell. The inner radius of the inner shell agrees well with the mean radius of $43^{\prime \prime}$ of the detached, geometrically thin CO gas shell of U Ant (Olofsson et al. 1996). The $\mathrm{CO}$ gas shell is probably the direct counterpart of the inner dust shell. The presence of clear outer and inner boundaries of the $\mathrm{CO}$ gas shell due primarily to the actual variation of mass-loss strongly supports that the inner dust shell is hollow and that the outer dust shell is detached from the inner shell. This double shell structure has been proposed for this star by Izumiura et al. (1997). Using the distance of $256 \mathrm{pc}$ (HIPPARCOS parallax of 3.90 milli-arcseconds, ESA 1997) we obtain mass-loss rates on the order of $10^{-6} \mathrm{M}_{\odot} \mathrm{yr}^{-1}$ for both shells, which is again consistent with the mass-loss rate derived for the CO gas 
shell (cf. Olofsson et al. 1996). Our ISOPHOT observations have revealed that U Ant underwent at least two high mass-loss phases, each of which was followed by a period of a significantly lower mass-loss, in the last $(1-2) \times 10^{4}$ years.

\section{Y CVn and RY Dra}

We found a very extended emission of $10^{\prime}$ diameter surrounding Y CVn at both $90 \mu \mathrm{m}$ and $160 \mu \mathrm{m}$. The brightness distribution is well reproduced by a hollow dust shell surrounding the central star. Main results were already published in the A\&A ISO special issue (Izumiura et al. 1996). With the PIA ver. 7.1 (Gabriel et al. 1997) the intensity calibration has been improved, which results in scaling up the brightness in the $90 \mu \mathrm{m}$ and $160 \mu \mathrm{m}$ maps by factors of 1.7 and 1.0, respectively. The model fitting gives a better power index of the dust opacity law of 1.1 instead of the previous value of 0.83 . The improved calibration, however, does not change significantly the shape of the dust shell, hence, little influences our previous conclusions. Taking into account the shell asymmetry, we obtained the inner radius of the shell as $150^{\prime \prime}-200^{\prime \prime}$ or $(4.9-6.5) \times 10^{17} \mathrm{~cm}$, and the mass-loss rate in the shell as (5-9) $\times 10^{-6} \mathrm{M}_{\odot} \mathrm{yr}^{-1}$ with the new calibration and the distance of 218 pc (HIPPARCOS parallax of 4.59 milli-arcseconds, ESA 1997).

RY Dra is the second brightest J-type carbon star next to Y CVn. We do not find, however, any obvious extended dust shell like the one seen in Y CVn. Since RY Dra is estimated to be 1.5-2 times more distant than Y CVn (Groenewegen et al. 1992; ESA 1997), it is possible that an extended dust shell is indeed present but is too close to the central star to be detected easily. Furthermore, cirrus confusion is significant for RY Dra, in particular at $160 \mu \mathrm{m}$, which indeed hampers detecting faint extended emission even if present. We have found that the $90 \mu \mathrm{m}$ image is well fitted with our model by adopting an extended dust shell of inner radius around $68^{\prime \prime}$. The mass-loss rate in the shell is found to be about $10^{-6} \mathrm{M}_{\odot} \mathrm{yr}^{-1}$. But the presence of the shell is not confirmed in the $160 \mu \mathrm{m}$ image because of the cirrus contamination. Therefore the presence of an extended dust shell is inconclusive in the present analysis. It is necessary to remove the influence of the cirrus confusion before we draw any conclusions about the presence of an extended dust shell around RY Dra.

\section{Some implications of the results}

Reliable HIPPARCOS parallaxes are available for U Ant and Y CVn among our program stars, and give distances of $256 \mathrm{pc}$ and $218 \mathrm{pc}$, respectively. The bolometric lumonosities of $\mathrm{U}$ Ant and $\mathrm{Y} \mathrm{CVn}$, then, become $5890 \mathrm{~L}_{\odot}$ and $5360 \mathrm{~L}_{\odot}$ (Groenewegen et al. 1992). The difference is smaller than the observational uncertainties. We therefore assume in the following discussion that the program stars have the same bolometric luminosity and are in similar evolutionary stages.

$\mathrm{R} \mathrm{Scl}$ has hollow dust and gas shells which are similar to the inner shells of $\mathrm{U}$ Ant. Since the distances to $\mathrm{R} \mathrm{Scl}$ and U Ant are also similar (Groenewegen et al. 1992), the shells of $R$ Scl are smaller than those of $U$ Ant in linear size, which probably means that the shells of $\mathrm{R} \mathrm{Scl}$ are younger than those of U Ant. Then, one would expect that another dust shell, somewhat smaller than the outer shell 
of U Ant, could be seen around R Scl if each dust shell formation has a 1 to 1 correspondence with the thermal pulse in the carbon star phase. However, we find that it is not the case. This implies that the formation of a hollow dust shell could be episodic having nothing to do with the thermal pulse, or that the thermal pulse could occur not very periodically. The latter case requires a period fluctuation as long as $10^{4}$ years. Otherwise the above assumptions are no longer valid and some differences in the evolutionary status or in the initial mass which cause a difference in the pulse period may be responsible for the difference in the mass-loss behavior of $\mathrm{U}$ Ant and $\mathrm{R} \mathrm{Scl}$.

In order to further elucidate the mass-loss behavior of individual stars on the AGB, much more observations with higher spatial resolutions as well as higher sensitivities in the far-infrared are required. Infrared Imaging Surveyor (IRIS), a Japanese infrared satellite which will be launched in 2003 , would greatly enrich the study of such mass-loss behaviors in individual stars.

Acknowledgments. This study is based on observations with ISO, an ESA project with instruments funded by ESA Member States with the participation of ISAS and NASA. PIA is a joint development by the ESA Astrophysics Division and the ISOPHOT Consortium led by the Max Planck Institute for Astronomy (MPIA), Heidelberg. Contributing ISOPHOT Consortium institutes are DIAS, RAL, AIP, MPIK, and MPIA.

\section{References}

ESA, 1997, The HIPPARCOS Catalogue, ESA SP-1200

Gabriel C., Acosta-Pulido J., Heinrichsen I., Skaley D., Morris H., Tai W.-M., 1997, in Astronomical Data Analysis Software and Systems VI, eds. G. Hunt \& H.E. Payne, ASP Conf. Ser. 125, p. 108

Groenewegen M.A.T., de Jong T., van der Bliek N.S., Slijkhuis S., Willems F.J., 1992, A\&A 253, 150

Hashimoto O., Izumiura H., 1997, Ap\&SS 251, 207

Izumiura H., Hashimoto O., Kawara K., Yamamura I., Waters L.B.F.M., 1996, A\&A 315, L221

Izumiura H., Waters L.B.F.M., de Jong T., Loup C., Bontekoe Tj.R., Kester D.J.M., 1997, A\&A 323, 449

Izumiura H., Waters L.B.F.M., de Jong T., Loup C., Hashimoto O., 1998, in The Carbon Star Phenomena, ed. R. Wing, IAU Symposium 177, in press

Kessler M.F., Steinz J.A., Anderegg M.E., et al., 1996, A\&A 315, L27

Klaas U., Krüger H., Heinrichsen I., Heske A., Laureijs R. (eds), 1994, ISOPHOT Observer's Manual, version 3.1

Lemke D., Klaas U., Abolins J., et al., 1996, A\&A 315, L64

Olofsson H., Bergman P., Eriksson K., Gustafsson B., 1996, A\&A 311, 587

van der Veen W.E.C.J., Habing H.J., 1988, A\&A 194, 125

Waters L.B.F.M., Loup C., Kester D.J.M., Bontekoe Tj.R., de Jong T., 1994, A\&A 281, L1 